\title{
NOUVELle
}

\section{La voie de signalisation du récepteur DAF-2 (Insuline/IGF-1) : un acteur clé du vieillissement musculaire}

Mathilde Solyga $^{1}$, Florence Solari ${ }^{2}$
> Un des enjeux actuels en santé publique est d'augmenter l'espérance de vie en bonne santé. Le vieillissement s'accompagne d'une diminution de la fonction et de la masse musculaire, phénomène également appelé sarcopénie. Chez le sujet âgé, la sarcopénie engendre des problèmes de dépendance et de fragilité [1]. Les études portant sur les mécanismes et les voies de signalisation impliquées dans le vieillissement sont souvent réalisées dans des modèles ayant une durée de vie courte. Le nématode Caenorhabditis elegans (C. elegans) dont la durée de vie est de deux semaines environ, est l'un des organismes de prédilection de ce type de recherche. Chez les mutants $C$. elegans daf-2-/- (orthologue unique chez le ver de la famille des récepteurs de l'insuline et de l'IGF-l présents chez les mammifères), la voie de signalisation de l'insuline est régulée négativement, ce qui entraine l'activation du facteur de transcription DAF-16/FOXO, qui est associée à un allongement de la durée de vie du ver. Cette augmentation de la longévité s'accompagne également d'une amélioration de la locomotion chez les vers âgés [2]. Les mécanismes impliqués dans le vieillissement peuvent varier selon les tissus. Pour le muscle, le
${ }^{1}$ M2 BMC parcours génétique de la cellule et pathologies, Université Claude Bernard Lyon 1, Lyon, France. mathilde.solyga@etu.univ-lyonl.fr ${ }^{2}$ INMG/CNRS UMR 5310/Inserm U1217/ Université Claude Bernard Lyon 1/Faculté de médecine et de pharmacie, Lyon, France. florence.solari@univ-lyonl.fr

rôle de voies de signalisation particulières, en particulier celle de l'Insuline/ IGF-1, n'était pas encore élucidé [3].

Les biomarqueurs du vieillissement musculaire

La perte de mobilité est le reflet du vieillissement musculaire au niveau fonctionnel. Cependant la mobilité dépend à la fois de l'intégrité du muscle mais aussi de celle de son innervation, d'où la nécessité de définir des marqueurs du vieillissement spécifiques du muscle. Une étude récente a montré que des changements musculaires surviennent précocement au cours de la vie adulte 


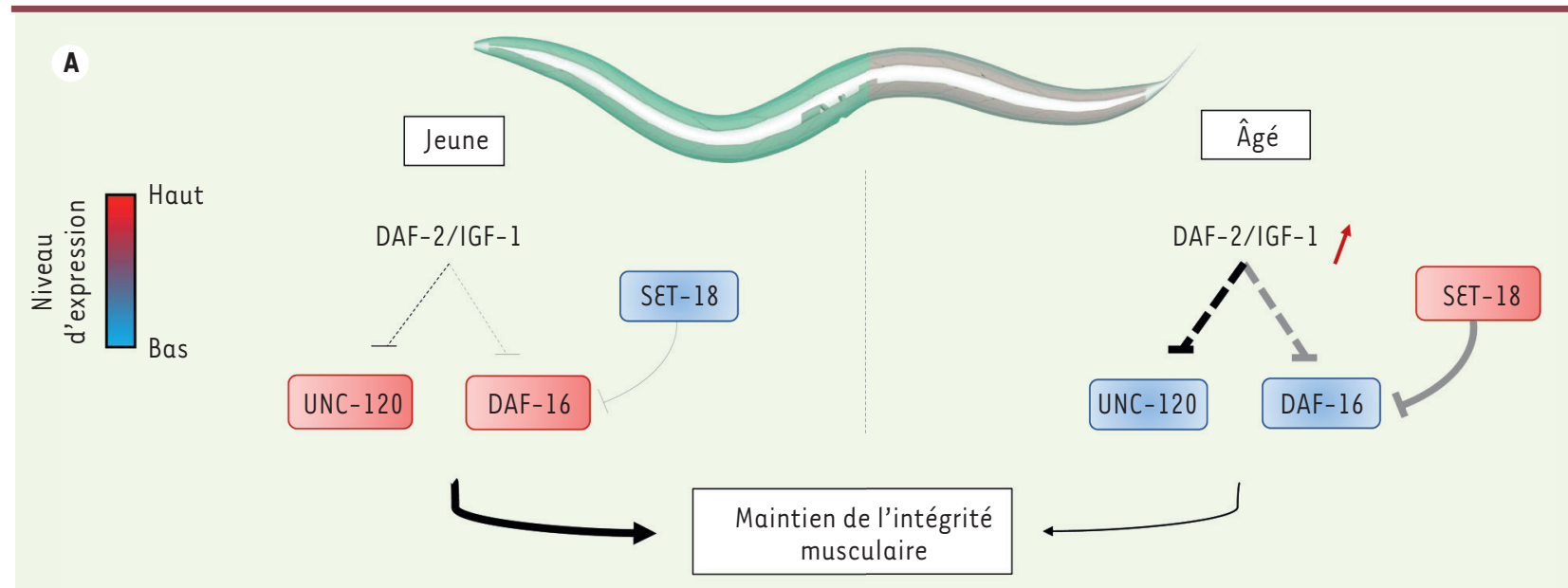

B

(1)

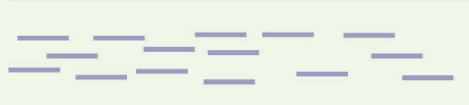

(2)

ares ans

ane
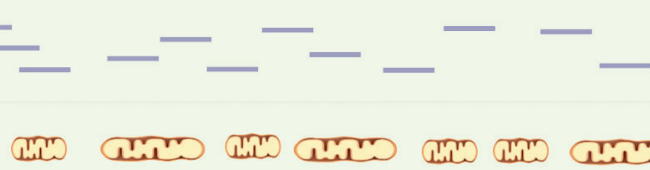

Vieillissement

(3)
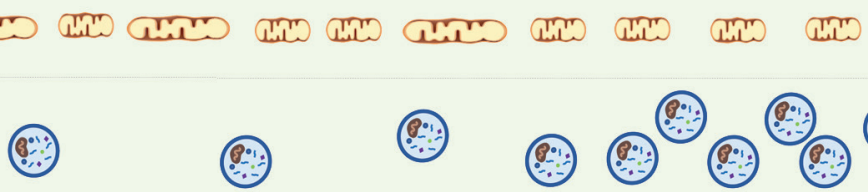

Figure 1. Modulation du vieillissement musculaire par la signalisation du récepteur DAF-2 chez C. elegans. A. Modulation génétique du vieillissement musculaire : la relation entre les acteurs moléculaires est indiquée par des connecteurs : interaction moléculaire directe (trait plein), interaction moléculaire indirecte (trait en pointillé). Interaction établie spécifiquement dans le muscle (trait noir), interaction établie mais pas spécifiquement dans le muscle (trait gris). L'épaisseur des traits est proportionnelle à l'activité des effecteurs moléculaires. Avec l'âge, l'activité de la signalisation du récepteur DAF-2 augmente [8] (flèche rouge). Les niveaux d'expression relatifs des acteurs moléculaires entre les individus jeunes et âgés sont indiqués en rouge (fortement exprimé) et en bleu (faiblement exprimé). $B$. Cinétique des changements musculaires avec l'âge : 1,2 et 3 correspondent aux marqueurs du vieillissement musculaire : (1) diminution de certains transcrits spécifiques du muscle ; (2) fragmentation progressive des mitochondries ; (3) augmentation du nombre de vésicules autophagiques.

de C. elegans [4]. Dès le premier jour de vie adulte, la transcription de certains gènes musculaires diminue de manière drastique sans pour autant affecter la mobilité. De même, une fragmentation mitochondriale dans les cellules musculaires est observée de façon très précoce à partir du deuxième jour de vie adulte durant le vieillissement. La fragmentation mitochondriale est, elle, corrélée à l'immobilité des vers, et est donc le reflet direct de la diminution de la fonction musculaire. À partir du $7^{\mathrm{e}}$ jour de vie adulte, on observe une accumulation de vésicules autophagiques survenant après la diminution des performances physiques. La diminution de l'expression des gènes et la fragmentation mitochondriale sont donc des marqueurs précoces du vieillissement musculaire et pourraient jouer un rôle causal dans la sarcopénie. Par contre, l'accumulation de vésicules autophagiques est un marqueur tardif et serait plutôt une conséquence du vieillissement [4] (Figure 1). Remarquablement, dans cette étude, le marquage endogène des filaments d'actine et de myosine n'a pas montré de défaut dans la structure des myofilaments, même à un âge lorsque plus de la moitié des vers sont immobiles. Ce résultat est en contradiction avec une étude précédente utilisant un transgène de fusion de la green fluorescent protein (GFP) avec la myosine, qui montre une désorganisation des myofilaments à un âge avancé [3]. Cependant, ce type de gène rapporteur ne permet pas de respecter les niveaux physiologiques d'expression des protéines, ce qui peut entrainer une certaine toxicité cellulaire et expliquer les différences observées entre les deux études.

\section{Les effecteurs transcriptionnels de la signalisation du récepteur DAF-2 dans le contrôle du vieillissement musculaire} L'étude des différents marqueurs du vieillissement a permis d'analyser plus en détail l'implication de différents acteurs de la voie DAF-2 dans le processus de vieillissement.

$\varepsilon n$ effet, les mutants daf-2 présentent un délai de l'apparition des marqueurs du vieillissement musculaire précédemment décrits, corrélé à une per- 
sistance des capacités physiques avec l'âge [4]. Le facteur de transcription DAF-16 est constitutivement activé chez les mutants daf-2. Le phénotype de mobilité de ces mutants requiert la fonction de DAF-16 dans le muscle, mais son activation dans d'autres tissus peut également influencer le vieillissement musculaire. En effet, la mobilité du double mutant daf-2/daf-16 est identique à celle des vers sauvages et seule la réexpression de daf-16 dans le muscle permet de restaurer le phénotype de mobilité des mutants daf- 2 . $\varepsilon$ n revanche, la fragmentation mitochondriale observée pendant le vieillissement musculaire peut être retardée via la ré-expression de DAF-16 dans le muscle ou le système nerveux des vers daf-2/daf-16 déficients, ce qui suggère une action différente de DAF-16 selon le tissu où il est exprimé [5]. Cependant l'implication de DAF-16 sur l'apparition des autres marqueurs du vieillissement musculaire, tels que la diminution de l'expression de certains gènes musculaires et l'accumulation de vésicules autophagiques reste à définir.

Le facteur de transcription UNC-120 (orthologue du « serum response factor » présent chez les mammifères) est un facteur impliqué dans le maintien de la fonction musculaire dont l'expression diminue avec l'âge [4]. Chez les mutants daf-2, l'expression de unc120 est maintenue au cours du temps par rapport à celle des vers contrôles, suggérant un rôle inhibiteur de DAF-2 sur l'expression de ce facteur de transcription. De plus, l'inhibition de l'expression de unc-120 grâce à des ARN interférents (ARNi) dans les mutants $d a f-2$, entraine une accélération $d u$ vieillissement musculaire sans impacter la longévité. De ce fait, il semblerait qu'UNC-120 soit un régulateur spécifique du vieillissement musculaire sous contrôle de la signalisation du récepteur DAF-2 [4].

Ces résultats montrent que la voie de signalisation du récepteur DAF-2 régule le vieillissement musculaire par l'inter- médiaire de la modulation d'au moins deux facteurs de transcription : DAF16/FOXO et UNC-120/SRF (Figure 1). II serait intéressant de déterminer si ces deux facteurs agissent de manière indépendante ou conjointe, notamment en identifiant les gènes cibles de ces deux facteurs de transcription.

\section{Rôle des facteurs épigénétiques dans le contrôle du vieillissement musculaire par DAF-2}

La protéine LIN-53 (orthologue des protéines RBBP4 et RBBP7 présentes chez les mammifères) est retrouvée dans différents complexes de remodelage de la chromatine, capables d'inhiber ou d'activer l'expression de gènes. L'interaction de LIN-53 avec le complexe SIN-3 promeut la longévité chez C. elegans en régulant le métabolisme. Chez les mutants daf-2, LIN-53 est nécessaire pour l'extension de la durée de vie et le maintien des taux de tréhalose (métabolite ayant un rôle bénéfique sur la longévité), ce qui suggère une coopération entre la voie DAF-2 et LIN53. L'inhibition d'expression de lin-53 par ARNi entraine une diminution de l'accumulation nucléaire de DAF-16, ce qui conforte l'hypothèse d'un lien entre LIN-53 et la signalisation de DAF-2.

LIN-53 agit aussi dans le complexe NuRD pour réguler le développement et le maintien de la fonction musculaire. $\varepsilon n$ effet, les mutants lin-53 présentent une désorganisation des fibres musculaires au stade larvaire et une surexpression d'UNC-120 [6]. Ceci est surprenant dans la mesure où UNC-120 promeut la fonction musculaire [4]. On peut penser que les défauts développementaux entrainés par la mutation lin-53 activent des mécanismes de compensation via UNC120 pour maintenir la fonction musculaire. À l'inverse, la surexpression de LIN-53 spécifiquement dans le muscle est suffisante pour améliorer la mobilité des vers plus âgés, sans affecter leur longévité.

Remarquablement, certains facteurs de remodelage de la chromatine sont exprimés spécifiquement dans certains tissus. Ainsi, l'expression de l'histone H3K36 diméthyltransférase SET-18 est limitée au muscle et augmente au cours du vieillissement. SET-18 régule négativement l'expression de DAF-16 via la méthylation de son promoteur et sa délétion allonge la durée de vie des vers. Néanmoins, le rôle de SET-18, plus spécifiquement dans le vieillissement musculaire, reste à déterminer (Figure 1) [7].

L'ensemble de ces données suggèrent fortement que LIN-53 et SET-18 contrôlent avec DAF-2 le vieillissement musculaire en agissant sur le facteur de transcription DAF-16/FOXO de façon directe (via la régulation de son expression) ou indirecte (via la régulation de sa localisation subcellulaire) [6].

\section{Conclusion}

Au cours du vieillissement, l'expression des facteurs de transcription DAF-16/FOXO et d'UNC-120/SRF diminue, entraînant une perte de l'intégrité musculaire qui peut être évaluée par l'analyse de marqueurs spécifiques du vieillissement musculaire. Par ailleurs, la régulation de la longévité par DAF-2 dépend de facteurs épigénétiques (SET-18, LIN-53) dont l'implication dans le vieillissement musculaire reste à préciser. II est aussi important de considérer le lien étroit entre les muscles et le système nerveux. En effet, La stimulation pharmacologique de la fonction nerveuse permet d'améliorer l'activité motrice chez le ver âgé [9]. Par ailleurs, l'exercice physique est connu pour son effet bénéfique sur le vieillissement musculaire chez différentes espèces; cependant, le rôle des récepteurs de l'insuline/IGF-l dans ce processus n'est pas établi. Chez C. elegans, il est possible de reproduire l'effet d'un effort physique par la pratique d'exercices de nage, ce qui offre l'opportunité d'analyser plus précisément le rôle potentiel de DAF-2 [10]. Enfin, la conservation au cours de l'évolution des effecteurs de DAF-2 laisse penser 
qu'ils pourraient être aussi impliqués dans le vieillissement musculaire chez les mammifères. Par exemple, la délétion de unc-120/SRF accélère le vieillissement musculaire chez la souris. De plus, de façon similaire à C. elegans, l'expression de unc-120/SRF diminue avec l'âge dans un modèle murin mais aussi chez l'homme [11]. $\diamond$

DAF-2 receptor signalling pathway (Insulin/IGF-1), a key role in muscular aging

\section{LIENS D'INTÉRÊT}

Les auteures déclarent n'avoir aucun lien d'intérêt concernant les données publiées dans cet article.

\section{RÉFÉRENCES}

1. Augustin H, Partridge L. Invertebrate models of agerelated muscle degeneration. Biochim Biophys Acta 2009 ; 1790 : 1084-94.

2. Uno $M$, Nishida $\varepsilon$. Lifespan-regulating genes in C. elegans. Npj Aging Mech Dis 2016 ; $2: 1-8$.

3. Herndon LA, Schmeissner PJ, Dudaronek JM, et al. Stochastic and genetic factors influence tissuespecific decline in ageing C. elegans. Nature 2002 ; 419: 808-14.

4. Mergoud Dit Lamarche A, Molin L, Pierson L, et al. UNC-120/SRF independently controls muscle aging and lifespan in Caenorhabditis elegans. Aging Cell $2018 ; 17$.

5. Wang $\mathrm{H}$, Webster $\mathrm{P}$, Chen $\mathrm{L}$, et al. Cell-autonomous and non-autonomous roles of daf- 16 in muscle function and mitochondrial capacity in aging C. elegans. Aging 2019; 11 : 2295-311.

6. Müthel S, Uyar B, He M, et al. The conserved histone chaperone LIN-53 is required for normal lifespan and maintenance of muscle integrity in Caenorhabditis elegans. Aging Cell 2019 ; el3012.

7. Su L, Li H, Huang C, et al. Muscle-specific histone H3K36 dimethyltransferase SET-18 shortens lifespan of Caenorhabditis elegans by repressing daf-16a expression. Cell Rep $2018 ; 22: 2716-29$.

8. Tawo R, Pokrzywa W, Kevei $\varepsilon$, et al. The ubiquitin ligase CHIP integrates proteostasis and aging by regulation of insulin receptor turnover. Cell $2017 ; 169: 470-82$. el3.

9. Liu J, Zhang B, Lei H, et al. Functional aging in the nervous system contributes to age-dependent motor activity decline in C. elegans. Cell Metab 2013; 18 : 392-402.

10. Laranjeiro R, Harinath G, Burke D, et al. Single swim sessions in $C$. elegans induce key features of mammalian exercise. BMC Biol 2017 ; 15 : 30.

11. Lahoute C, Sotiropoulos A, Favier M, et al. Premature aging in skeletal muscle lacking serum response factor. PLoS One 2008 ; 3 . 